$\square$ Math-Net.Ru

Общероссийский математический портал

А. И. Козко, А. В. Рождественский, О неравенстве Джексо- 
на с обобщенным модулем непрерывности, Матем. заметкu, 2003, том 73, выпуск 5, 783-788

DOI: https://doi.org/10.4213/mzm632

Использование Общероссийского математического портала Math-Net.Ru подразумевает, что вы прочитали и согласны с пользовательским соглашением http://www. mathnet.ru/rus/agreement

Параметры загрузки:

IP : 54.197 .130 .99 
26 апреля 2023 г., 14:44:24

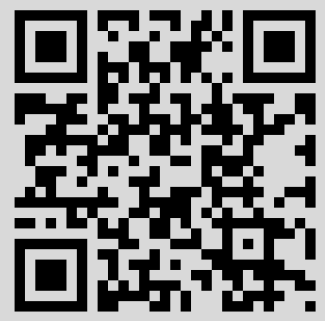




\section{O НЕРАВЕНСТВЕ ДЖЕКСОНА С ОБОБЩЕННЫМ МОДУЛЕМ НЕПРЕРЫВНОСТИ}

\section{А. И. Козко, А.В. Рождественский}

Следуя работам Шапиро и Бомана [1]-[3], обобщенным модулем непрерыв вности функции $f \in L_{2}\left(\mathbb{T}^{d}\right), d \in \mathbb{N}$, будем назьвать величину

$$
\bar{\omega}_{\psi}(f, T)=\max _{t \in T}\left(\sum_{s} \psi(s t)\left|\hat{f}_{s}\right|^{2}\right)^{1 / 2} .
$$

Здесь $\psi(\cdot)$-заданная непрерьвная неотрицательая $2 \pi$-периодическая функция, $\psi(0)=0, \psi(\cdot) \not \equiv 0$, а $T$ - заданное замкнутое подмножество $[0,2 \pi]^{d}$.

Работа первого автора вьполнена при поддержке Российского фонда фундаментальных исследований, грант № 02-01-00248, и INTAS, грант № 99-01080, а второго автора - при поддержке Российского фонда фундаментальных исследований, гранты № 00-15-96109 и № 02-01-00248.

(C) А. И. Козко, А. В. РОЖДЕСтвенСКий 
Пусть $\Lambda$ - собственное подмножество $\mathbb{Z}^{d}$, содержащее начало координат. Через $E_{\Lambda}(f)=E_{\Lambda}(f)_{2}$ обозначим величину наилучшего приближения элемента $f \in L_{2}\left(\mathbb{T}^{d}\right)$ функциями, спектр которых сосредоточен на множестве $\Lambda$.

Неравенством Джексона с обобщенным модулем непрерывности назовем следующее соотношение:

$$
E_{\Lambda}(f) \leqslant C \bar{\omega}_{\psi}(f, T) \quad \forall f \in L_{2}\left(\mathbb{T}^{d}\right),
$$

где константа $C$ не зависит от выбора функции $f$. Точную нижнюю грань таких констант назовем точной константой в неравенстве Джсексона с обобщенным модулем непрерывности и обозначим через $\varkappa_{\psi}^{(d)}(\Lambda, T)$.

Первый вопрос, на который мы даем ответ в данной работе: какова величина минимальной точной константы в неравенстве (1) (под которой нам представляется естественным понимать величину $\left.\varkappa_{\psi}^{(d)}\left(\Lambda, \mathbb{T}^{d}\right)\right) ?^{1}$

Теорема 1. Пусть множество $\Lambda \subset \mathbb{Z}^{d}$ конечно и содержит начало координат. Тогда минимальная точная константа в неравенстве (1) равна

$$
\varkappa_{\psi}^{(d)}\left(\Lambda, \mathbb{T}^{d}\right)=\left(\frac{1}{2 \pi} \int_{0}^{2 \pi} \psi(x) d x\right)^{-1 / 2} .
$$

ДоКАЗАТЕЛЬСТво. Используя формулу двойственности для расстояния от точки 0 до вьпуклого множества $A$ в нормированном пространстве $X=C\left(\mathbb{T}^{d}\right)\left(\right.$ см. [4]), $\inf \left\{\|g\|_{X}: g \in A\right\}=$ $\max \left\{\inf _{g \in A}\left\langle g^{*}, g\right\rangle: g^{*} \in X^{*},\left\|g^{*}\right\|_{X^{*}} \leqslant 1\right\}$, получим двойственное представление точной константы в неравенстве Джексона:

$$
\varkappa_{\psi}^{(d)}\left(\Lambda, \mathbb{T}^{d}\right)=\left(\max _{\mu \in B \mathcal{M}^{+}\left(\mathbb{T}^{d}\right)} \inf _{k \notin \Lambda} \int_{\mathbb{T}^{d}} \psi(k t) d \mu(t)\right)^{-1 / 2},
$$

где $B \mathcal{M}^{+}\left(\mathbb{T}^{d}\right)$ - множество конечных борелевских неотрицательных мер на $\mathbb{T}^{d}$, норма которых не превосходит 1 .

Пусть $K=\sup \left\{k_{1}: k=\left(k_{1}, \ldots, k_{d}\right) \in \Lambda\right\}$. Тогда

$$
\inf _{k \notin \Lambda} \int_{[0,2 \pi]^{d}} \psi(k t) d \mu(t) \leqslant \inf _{k_{1}>K, k_{1} \in \mathbb{Z}} \int_{[0,2 \pi]^{d}} \psi\left(k_{1} t_{1}\right) d \mu(t) .
$$

Представим $\int_{[0,2 \pi]^{d}} \psi\left(k_{1} t_{1}\right) d \mu(t)$ в виде суммы

$$
\int_{[0,2 \pi]^{d}} \psi\left(k_{1} t_{1}\right) d \mu(t)=\int_{\Omega_{1}} \psi\left(k_{1} t_{1}\right) d \mu(t)+\int_{\Omega_{2}} \psi\left(k_{1} t_{1}\right) d \mu(t),
$$

где $\Omega_{1}=\left\{t \in[0,2 \pi]^{d}: t_{1} / \pi \in \mathbb{Q}\right\}, \Omega_{2}=[0,2 \pi]^{d} \backslash \Omega_{1}$.

Заф̆иксируем произвольное $\epsilon>0$ и выберем натуральное $Q$ такое, что

$$
\int_{\Omega_{1}} \psi\left(l Q t_{1}\right) d \mu(t) \leqslant \epsilon \quad \forall l \in \mathbb{N}
$$

Тогда

$$
\begin{aligned}
\inf _{l \in \mathbb{N}} \int_{[0,2 \pi]^{d}} \psi\left(l Q t_{1}\right) d \mu(t)= & \inf _{l \in \mathbb{N}}\left(\int_{\Omega_{1}} \psi\left(l Q t_{1}\right) d \mu(t)+\int_{\Omega_{2}} \psi\left(l Q t_{1}\right) d \mu(t)\right) \\
& \leqslant \epsilon+\inf _{l \in \mathbb{N}} \int_{\Omega_{2}} \psi\left(l Q t_{1}\right) d \mu(t)=\epsilon+\inf _{N \in \mathbb{N}} \min _{1 \leqslant l \leqslant N} \int_{\Omega_{2}} \psi\left(l Q t_{1}\right) d \mu(t) .
\end{aligned}
$$

${ }^{1}$ Очевидно, что если $A \subset B, A, B$ - замкнутые множества, то $\varkappa_{\psi}^{(d)}(\Lambda, A) \geqslant \varkappa_{\psi}^{(d)}(\Lambda, B)$. 
Введем функцию $\bar{\psi}_{N}(x):=\frac{1}{N} \sum_{l=1}^{N} \psi(l x)(\forall x \in \mathbb{R})$. Нетрудно видеть, что

$$
\min _{1 \leqslant l \leqslant N} \int_{\Omega_{2}} \psi\left(l Q t_{1}\right) d \mu(t) \leqslant \frac{1}{N} \sum_{l=1}^{N} \int_{\Omega_{2}} \psi\left(l Q t_{1}\right) d \mu(t)=\int_{\Omega_{2}} \bar{\psi}_{N}\left(Q t_{1}\right) d \mu(t) .
$$

Так как при каждом $t_{1} \in[0,2 \pi] \backslash 2 \pi \mathbb{Q}$ последовательность $\left\{l \cdot Q t_{1}\right\}_{l=1}^{\infty}$ равномерно распределена на $\mathbb{T}$, по критерию Вейля $[5$, c. 82$]$

$$
\lim _{N \rightarrow \infty} \bar{\psi}_{N}\left(Q t_{1}\right)=\frac{1}{2 \pi} \int_{0}^{2 \pi} \psi(x) d x \quad \forall t_{1} \notin 2 \pi \mathbb{Q} .
$$

При этом $\left|\bar{\psi}_{N}\left(Q t_{1}\right)\right| \leqslant \max _{1 \leqslant l \leqslant N}\left|\psi\left(l Q t_{1}\right)\right| \leqslant\|\psi(\cdot)\|_{C(\mathbb{T})}$, т.е. $\left|\bar{\psi}_{N}\left(Q t_{1}\right)\right|$ равномерно ограничено по $t_{1}$ и $N$. Поэтому по теореме Лебега о предельном переходе

$$
\lim _{N \rightarrow \infty} \int_{\Omega_{2}} \bar{\psi}_{N}\left(Q t_{1}\right) d \mu(t)=\int_{\Omega_{2}}\left(\frac{1}{2 \pi} \int_{0}^{2 \pi} \psi(x) d x\right) d \mu(t) \leqslant \frac{1}{2 \pi} \int_{0}^{2 \pi} \psi(x) d x .
$$

Следовательно,

$$
\begin{aligned}
\inf _{k \geqslant K+1, k \in \mathbb{N}} \int_{[0,2 \pi]^{d}} \psi(k t) d \mu(t) & \leqslant \inf _{l \in \mathbb{N}} \int_{[0,2 \pi]^{d}} \psi\left(l Q t_{1}\right) d \mu(t) \\
& \leqslant \epsilon+\lim _{N \rightarrow \infty} \int_{\Omega_{2}} \bar{\psi}_{N}\left(Q t_{1}\right) d \mu(t) \\
& \leqslant \epsilon+\frac{1}{2 \pi} \int_{0}^{2 \pi} \psi(x) d x .
\end{aligned}
$$

Отсюда, учитывая произвол в выборе $\epsilon>0$, заключаем, что

$$
\inf _{k \notin \Lambda} \int_{[0,2 \pi]^{d}} \psi(k t) d \mu(t) \leqslant \inf _{k \geqslant K+1, k \in \mathbb{N}} \int_{[0,2 \pi]^{d}} \psi\left(k t_{1}\right) d \mu(t) \leqslant \frac{1}{2 \pi} \int_{0}^{2 \pi} \psi(x) d x
$$

для любой меры $\mu \in B \mathcal{M}^{+}\left(\mathbb{T}^{d}\right)$.

С другой стороны,

$$
\max _{\mu \in B \mathcal{M}^{+}\left(\mathbb{T}^{d}\right)} \inf _{k \notin \Lambda} \int_{[0,2 \pi]^{d}} \psi(k t) d \mu(t) \geqslant \inf _{k \notin \Lambda} \int_{[0,2 \pi]^{d}} \psi(k t) \frac{d t}{(2 \pi)^{d}} .
$$

А в силу $2 \pi$-периодичности $\psi(\cdot)$ для всякого $k \in \mathbb{Z}^{d}, k \neq 0$, справедливо равенство

$$
\int_{[0,2 \pi]^{d}} \psi(k t) \frac{d t}{(2 \pi)^{d}}=\frac{1}{2 \pi} \int_{0}^{2 \pi} \psi(x) d x .
$$

Теорема доказана.

Приведем следствие из этой теоремы, относящееся к неравенству Джексона с "классическим" модулем произвольного порядка (которое еще принято назьвать неравенством ДжсексонаСтечкина), усиливающее результаты работ [6]-[8].

Для этого сначала заметим, что классический модуль непрерывности порядка $r$ в пространстве $L_{2}\left(\mathbb{T}^{d}\right)$

$$
\omega_{r}(f, T)_{2}=\sup _{t \in T}\left\|\Delta_{t}^{r} f\right\|_{2},
$$

совпадает с обобщенным модулем непрерывности, отвечающим функции $\psi_{r}(x):=4^{r} \sin ^{2 r}(x / 2)$. При этом, $\int_{0}^{2 \pi} \psi_{r}(x) d x=C_{2 r}^{r}$. (Здесь $\Delta_{t}^{r} f(\tau)=\Delta_{t}\left(\Delta_{t}^{r-1} f(\tau)\right), \Delta_{t} f(\tau)=\Delta_{t}^{1} f(\tau)=f(\tau+t)-$ $f(\tau), \Delta_{t}^{0} f(\tau)=f(\tau)$.) 
СлЕДСтвиЕ 1.1. В предполохении теоремы 1 относительно мнохества $\Lambda$ минимальная точная константа в неравенстве Джексона-Стечкина

$$
E_{\Lambda}(f) \leqslant C \omega_{r}(f, T) \quad \forall f \in L_{2}\left(\mathbb{T}^{d}\right)
$$

равна $1 / \sqrt{C_{2 r}^{r}}$.

Отметим, что в одномерном случае наиболее исследованньм является вопрос о поведении точной константы в неравенстве Джексона с модулем непрерьвности первого порядка. В работе [9] доказано, что точная константа в неравенстве ${ }^{2}$

$$
E_{n-1}(f)_{2} \leqslant C \omega(f, \delta)_{2} \quad \forall f \in L_{2}(\mathbb{T})
$$

равна $1 / \sqrt{2}$ при всех $\delta \geqslant \pi / n$.

Следующая проблема, на которую мы обращаем внимание, состоит в том, чтобы выделить класс обобщенных одномерных модулей непрерьвности, которые, во-первых, обладают тем же свойством стабилизации точной константы в неравенстве Джексона с $\delta=\pi / n$, как у классического модуля непрерьвности первого порядка, во-вторых, аннулируют множество алгебраических многочленов заданной степени $r-1$, в-третьих, удобны для вычислений, и, в-четвертых, - чтобы в случае, когда обобщенный модуль непрерывности задается при помощи разностного оператора (как, например, в классической ситуации), отношение величины минимальной точной константы в неравенстве Джексона с таким модулем к операторной норме соответствующего разностного операторамало отличалось (по порядку) от такого отношения для классического модуля непрерьвности порядка $r$.

Символом $\Upsilon$ обозначим класс непрерывных неотрицательных $\pi$-периодических четных функций. Для $\xi \in \Upsilon$ положим $h_{\xi}(x):=-\int_{0}^{\pi} \cos (x t) \xi(x t) \sin t d t$.

Пусть $\mathbf{b}=\left\{b_{k}\right\}$ - заданный конечный набор положительных чисел. Обозначим через $\Psi(\mathbf{b})$ множество четных непрерывных неотрицательных $2 \pi$-периодических функций $\psi(\cdot), \psi(0)=0, \psi(\cdot) \not \equiv 0$, для которых справедливо представление

$$
\int_{0}^{\pi} \psi(x t) \frac{\sin t}{2} d t=\frac{1}{2 \pi} \int_{0}^{2 \pi} \psi(t) d t+\sum_{k} h_{\xi_{k}}\left(b_{k} x\right) \quad \forall x \geqslant \hat{b}:=\max _{k} \frac{1}{b_{k}}
$$

с некоторьми $\xi_{k}(\cdot) \in \Upsilon$.

ТЕОРема 2. Пусть $\mathbf{b}=\left\{b_{k}\right\}-$ конечный набор положительных чисел, $\psi \in \Psi(\mathbf{b}), n \in \mathbb{N}$. Тогда точная константа в неравенстве

$$
E_{n-1}(f)_{2} \leqslant C \bar{\omega}_{\psi}(f, \delta) \quad \forall f \in L_{2}(\mathbb{T})
$$

равна $\left(\frac{1}{2 \pi} \int_{0}^{2 \pi} \psi(x) d x\right)^{-1 / 2}$ при всех $\delta \geqslant \min \left\{\frac{\pi}{n} \hat{b}, \pi\right\}$.

ДокАЗАТЕльСтво. Из теоремы 1 следует, что точная константа $\bar{\varkappa}_{\psi}(n, \delta)$ в неравенстве (3) ограничена снизу величиной $\left(\frac{1}{2 \pi} \int_{0}^{2 \pi} \psi(x) d x\right)^{-1 / 2}$. Чтобы получить такую же оценку сверху для точной константы, опять воспользуемся ее двойственным представлением, вследствие которого

$$
\bar{\varkappa}_{\psi}(n, \delta) \leqslant\left(\max _{\mu \in B \mathcal{M}^{+}([0, \pi])} \inf _{x \geqslant \hat{b}} \int_{0}^{\pi} \psi(x t) d \mu(t)\right)^{-1 / 2} .
$$

Учитывая, что $\int_{0}^{\pi} \frac{\sin t}{2} d t=1$ и $\sin t \geqslant 0(\forall t \in[0, \pi])$, полагая $d \mu(t)=\frac{\sin t}{2} d t$, получаем неравенство

$$
\max _{\mu \in B \mathcal{M}^{+}([0, \pi])} \inf _{x \geqslant \hat{b}} \int_{0}^{\pi} \psi(x t) d \mu(t) \geqslant \inf _{x \geqslant \hat{b}} \int_{0}^{\pi} \psi(x t) \cdot \frac{\sin t}{2} d t .
$$

\footnotetext{
${ }^{2}$ Здесь $E_{n-1}:=E_{\{-n+1, \ldots, n-1\}}$.

${ }^{3}$ Здесь, по аналогии с классическими обозначениями, $\bar{\omega}_{\psi}(f, \delta):=\bar{\omega}_{\psi}(f,[0, \delta])$.
} 
Следовательно, в силу представления (2) для того, чтобы установить справедливость неравенства $\bar{\varkappa}_{\psi}(n, \delta) \leqslant\left(\frac{1}{2 \pi} \int_{0}^{2 \pi} \psi(x) d x\right)^{-1 / 2}$ при всех $\delta \geqslant \pi \hat{b} / n$, достаточно доказать следующее свойство:

$$
h_{\xi}(x) \geqslant 0 \quad \forall x \geqslant 1, \forall \xi \in \Upsilon .
$$

Заметим, что функция $h_{\xi}(x)$ может быть представлена в виде $h_{\xi}(x)=g_{\xi}(x)+R_{\xi}(x)$, где

$$
g_{\xi}(x)=2 \cos \left(\frac{\pi}{2 x}\right) \int_{0}^{\pi / 2 x} \xi(x t) \cos (x t) \cdot \sin \left(\frac{\pi}{2 x}-t\right) d t
$$

если $1 \leqslant x \leqslant 2, l \in \mathbb{N}_{\text {и }}$

$$
g_{\xi}(x)=2 \cos ^{2}\left(\frac{(2 l+1) \pi}{2 x}\right) \int_{0}^{\pi / 2 x} \xi(x t) \cos (x t) \cdot \cos t \cdot\left(\operatorname{tg} \frac{\pi}{2 x}-\operatorname{tg} t\right) d t
$$

если $2 l \leqslant x \leqslant 2 l+2, l \in \mathbb{N}, \mathrm{a}$

$$
R_{\xi}(x)=\left\{\begin{array}{l}
2 \cos \frac{(2 l+1 / 2) \pi}{x} \int_{\pi}^{(2 l+1 / 2) \pi / x} \xi(x t) \cos (x t) \cdot \sin \left(t-\frac{(2 l+1 / 2) \pi}{x}\right) d t \\
\quad+\int_{(4 l+1-x) \pi / x}^{(2 l+1) \pi / x} \xi(x t) \cos (x t) \cdot \sin t d t \quad \text { при } \quad x \in[2 l, 2 l+1 / 2], \quad l \in \mathbb{N}, \\
\int_{\pi}^{(2 l+1) \pi / x} \xi(x t) \cos (x t) \cdot \sin t d t \quad \text { при } x \in[2 l+1 / 2,2 l+1], \quad l \in \mathbb{N}, \\
-\int_{(2 l+1) \pi / x}^{\pi} \xi(x t) \cos (x t) \cdot \sin t d t \quad \text { при } x \in[2 l+1,2 l+3 / 2], \quad l \in \mathbb{Z}_{+}, \\
2 \cos \frac{(2 l+3 / 2) \pi}{x} \int_{(2 l+3 / 2) \pi / x}^{\pi} \xi(x t) \cos (x t) \cdot \sin \left(\frac{(2 l+3 / 2) \pi}{x}-t\right) d t \\
\quad-\int_{(2 l+1) \pi / x}^{(4 l+3-x) \pi / x} \xi(x t) \cos (x t) \cdot \sin t \quad \text { при } x \in[2 l+3 / 2,2 l+2], \quad l \in \mathbb{Z}_{+} .
\end{array}\right.
$$

Нетрудно показать, что функции $g_{\xi}(x)$ и $R_{\xi}(x)$ неотрицательны при $x \geqslant 1$. Теорема доказана.

Пусть $r, a \in \mathbb{N}$. Обобщенным модулем Туэ-Морса функции $f \in L_{2}(\mathbb{T})$ назовем величину

$$
\widetilde{\omega}_{a, r}(f, \delta):=\max _{0 \leqslant t \leqslant \delta}\left\|\prod_{k=0}^{r-1} \Delta_{a^{k} t} f\right\|_{2}
$$

Ясно, что $\widetilde{\omega}_{a, r}(f, \delta)=\bar{\omega}_{\psi_{a, r}}(f,[0, \delta])$, где $\psi_{a, r}(x)=4^{r} \prod_{k=0}^{r-1} \sin ^{2}\left(\frac{a^{k}}{2} t\right)$.

СледСтвие 2.1. Пусть $a=2 s, s, n \in \mathbb{N}$. Тогда точная константа в неравенстве

$$
E_{n-1}(f)_{2} \leqslant C \widetilde{\omega}_{a, r}(f, \delta) \quad \forall f \in L_{2}(\mathbb{T})
$$

равна $1 / \sqrt{2^{r}}$ при всех $\delta \geqslant \pi / n$.

ДокАЗАТЕЛЬство. Нетрудно видеть, что $\frac{1}{2 \pi} \int_{0}^{2 \pi} \psi_{a, r}(x) d x=2^{r}$. Покажем, что функция $\psi_{a, r}(\cdot)$ принадлежит классу $\Psi(\mathbf{b})$ для $\mathbf{b}=\left(b_{0}, \ldots, b_{r-1}\right):=\left(a^{r-1}, \ldots, a^{0}\right)$. Действительно, положим $\xi_{0}(x)=2^{r-1}, \xi_{k}(x)=2^{r-1} \prod_{s=1}^{k}\left(1-\cos \left(a^{s} x\right)\right), k=1, \ldots, r-1$. Ясно, что $\xi_{k}(\cdot) \in \Upsilon$ и

$$
\int_{0}^{\pi} \psi_{a, r}(x t) \cdot \frac{\sin t}{2} d t=2^{r}+\sum_{k=0}^{r-1} h_{\xi_{k}}\left(a^{r-1-k} x\right) .
$$

Применяя теперь теорему 2 , получаем требуемое утверждение. 


\section{СПИСОК ЦИТИРОВАННОЙ ЛИТЕРАТУРЫ}

1. Shapiro H. S. // Acta Math. 1968. V. 120. P. 279-292. 2. Boman J., Shapiro H. S. // Arkiv för Matematik. 1971. V. 9. № 1. P. 91-116. 3. Boman J. // Arkiv för Matematik. 1980. V. 18. №1. Р. 73-100. 4. Иоффе А. Д., Тихомиров В. М. // УМН. 1968. Т. 23. №6. С. 51-116. 5. Касселс Дж. В.С. Введение в теорию диофантовых приближений. М.: ИЛ, 1961. 6. Бердышева Е. Е. // Матем. заметки. 1999. Т. 66. № 3. С. 336-350. 7. Черных Н. И. // Матем. заметки. 1967. Т. 2. №5. С. 513-522. 8. Виноградов О. Л., Жук В. В. // Проблемы матем. анализа. 2001. № 22. С. 3-26. 9. Черных Н. И. // Тр. МИАН. 1967. Т. 88. С. 71-74.

Московский государственный университет им. М. В. Ломоносова 